\title{
Time to Move Forward for Image Enhanced Endoscopy even in Resource Limited Areas
}

\author{
Mariam Zaghloul \\ Hepatology, Gastroenterology and Infectious Diseases Department.
} Faculty of Medicine, Kafrelsheikh University, Egypt

Mobile:

$+201205443335$

E mail: mariam_zaghloul@med.k fs.edu.eg

Key words: Image-enhanced endoscopy,

Chromoendoscopy, Narrow-band imaging, $i$ scan

\section{INTRODUCTION}

Providing high quality endoscopic services is now the unified language of all gastrointestinal societies. Endoscopy imaging of the gastrointestinal (GI) tract has evolved tremendously over the last few decades. Despite the revolutionary progress made to enhance the resolution of white-light endoscopy, early characterization of premalignant lesions is still challenging. The development of endoscopic images passed through different steps starting by using dyes, magnification endoscopy (ME), high-definition endoscopy, and image enhancement endoscopy (IEE) that we will be summarize here in short.

Historically, the fiber-optic endoscopy was first introduced in 1957 by Hirchowitz et al. to visually inspect the inside of the gastrointestinal tract [1]. Then fiber-optic scopes were developed to video scopes in the 1990s using charge-coupled devices (CCDs), an array of individual photocells, known as picture elements or pixels that transmit light reflected on the mucosal surface as electrons to the video processor to be reconverted into a digital image. The resolution of images relies upon the number of pixels in the array, with higher the pixel density providing a higher resolution for detailed images of a lesion [1]. This is the basic difference between standard and high definition endoscopes introduced in the early 2000s where standard definition endoscopes are equipped with CCD chips that produce image signals with a resolution of 100,000 to 400,000 pixels while those of the high- resolution endoscopes (HD) could reach million pixels leading to magnification effect up to a 35 -fold from the standard definition endoscopes [2].

For a clearer detailed image of subtle mucosal lesions and better characterization of early GI malignancies, magnification alone was not enough. Optical enhancements technologies was needed.

\section{IMAGE-ENHANCED}

ENDOSCOPY (IEE) have revolutionized the diagnosis of gastrointestinal disorders. IEE provides high-contrast images of lesions by means of optical or electronic technologies to augment enhancements of the mucosal surface and of blood vessels. IEE could be classified into: dye-based chromoendoscopy (old), digital methods, and optical-digital methods[3] e.g. chromoendoscopy, narrow-band imaging, i-SCAN, flexible spectral imaging color enhancement (FICE) and blue-light laser imaging.

\section{CHROMOENDOSCOPY}

(CE)

consists of spray application of dyes, which are harmless to the human body, onto the mucosal surface of interest. Stains are classified according to their mechanism of action into absorptive (or vital) stains, contrast stains and reactive stains. The application of dye improves visualization of the microstructure and vascular patterns of lesions under investigation. Thus improving 
outcomes in the screening of malignant and premalignant lesions [4]. Despite its significant advantage in detection and characterization, dye based IEE is not widely practiced as it is deemed to be time-consuming. Furthermore, certain dyes (e.g. crystal violet) are not readily available in many countries.

\section{ELECTRONIC CHROMOENDOSCOPY}

The new image processors could do image enhancement by converting color and structurebased diagnostic information into more objective and quantitative indicators. Electronic $\mathrm{CE}$ minimizes the needed time for spraying and suctioning of dyes and avoids the solution pooling in depressed-type lesions hindering visualization [5]. Various electronic $\mathrm{CE}$ are currently used, including the NBI, which selectively transmits a specific range of light using an optical filter, and the i-SCAN and FICE systems, which implement this process using software-driven post-image processing.

Narrow-band imaging (NBI) was first developed by Olympus Medical Systems using the basic physical principle that the depth of light penetration into a tissue is proportional to the wavelength used: blue bandwidth for superficial tissues, red bandwidth for deep tissue, and green bandwidth for intermediate tissues [6]. The blue shortwave visible light penetrates only superficially into the mucosa; it is not reflected as it is absorbed by the hemoglobin of the red blood cells in blood vessels. So, early GI cancer that arises in the mucosa with its suppling blood vessels could be easily differentiated from the normal vascular pattern of normal tissue [7]. The combined use of NBI with ME in the latest scopes has improved the clarity and accuracy of assessment of mucosal microvasculature and microstructure e.g. pitting surfaces of colon polyps and boundaries of Barrett's esophagus.

i-SCAN is a software-based digital postprocessing image-enhanced technology developed by PENTAX (Tokyo, Japan). i-SCAN combines three functions: surface enhancement (SE), contrast enhancement (CE), and tone enhancement (TE). The SE enhances light-dark contrast on the basis of luminance intensity data obtained from each pixel comparable to the structure enhancement feature of Olympus endoscopes (Tokyo, Japan) [8].

\section{Flexible spectral imaging color enhancement (FICE) and the blue laser imaging (BLI)}

FICE is a digital imaging post-processing system of Fujinon (Tokyo, Japan). FICE enhances the visualization of mucosal structure and microcirculation by the selection of spectral transmittance with a dedicated wavelength. The FICE system has been shown to be limited in producing high-contrast images of the microvasculature on the mucosal surface [9].

The FICE systems were replaced by the blue laser imaging (BLI) system (Fujifilm Co., Kanagawa, Japan) allowing the system to produce white-light images as well as NBI, based on the properties of laser light. Compared to the conventional xenon lamp source, laser light also has low power consumption and extremely low heat generation to identify subtle changes in the mucosal surface and early cancer diagnosis.

Ibrahim et al., in a study published in the current issue of the in Afro-Egypt J Infect Endem Dis entitled "Role of I-scan in Diagnosis of Duodenal Mucosal Lesions during Gastroduodenoscopy" gives us an example of how simple implementation of new IEE technologies would add to daily endoscopy practice and improve work outcomes. They evaluated the use of i-scan as an IEE in the evaluation and histological confirmation of mucosal lesions in the duodenum and its role in biopsy targeting. They examined 41 patients indicated for duodenal mucosa histological examination for undiagnosed iron deficiency anemia cause, progressive weight loss, chronic diarrhea or persistent abdominal pain. They compared examination under the conventional white light endoscopy and i-Scan imaging to the definitive diagnosis determined by histopathology examination of the biopsied specimen.

Interestingly, they showed that the criterionrelated validity of i-scan as a diagnostic test was 91.9\% sensitivity, $75 \%$ specificity, $97.1 \%$ PPV and 50\% NPV compared to white light endoscopy, which achieved $67.6 \%$ sensitivity, 75\% specificity, 96\% PPV and 20\% NPV. I-scan achieved $95.1 \%$ accuracy, $97 \%$ specificity, $75 \%$ sensitivity in the diagnosis of celiac disease and detection of duodenal villous atrophy compared to white light endoscopy, which achieved $92.7 \%$ accuracy, $100 \%$ specificity, $25 \%$ sensitivity. There was a significant association between disease diagnosis by I-scan and presenting by 
abdominal pain $(p<0.026)$. Similar studies showed that i-scan is useful in evaluation of duodenal villous atrophy [10] as well as acceptable performance in colonic polyps [11].

To conclude, proper early detection of GI lesions is the key factor for improving patients' prognosis and improving quality of care. It is our duty as a gastroenterologist offering endoscopic screening and diagnostic services to do the best for our patients care. In clinical studies, IEE outperformed conventional white-light endoscopy in diagnosing lesions and identifying their morphologies. Large-scale studies on various lesions for standardization of findings and training are needed. It is also hoped that every center could adopt the technology of IEE for better outcomes and quality.

\section{REFERENCES}

1. Subramanian, V, K. Ragunath, Advanced endoscopic imaging: a review of commercially available technologies. Clin Gastroenterol Hepatol 2014; 12(3): 368-76.e1.

2. ASGE Technology Committee, Kwon RS, Adler DG, Chand B, Conway JD, Diehl DL, et al. Highresolution and high-magnification endoscopes. Gastrointest Endosc 2009; 69(3 Pt 1):399-407.

3. Tajiri, H., H. Niwa, Proposal for a consensus terminology in endoscopy: how should different endoscopic imaging techniques be grouped and defined? Endoscopy 2008; 40(9): 775-8.

4. Peitz U, Malfertheiner P. Chromoendoscopy: from a research tool to clinical progress. Dig Dis 2002; 20(2): 111-9.
5. Jang J.-Y. The Past, Present, and Future of ImageEnhanced Endoscopy. Clinical Endoscopy 2015; 48(6): 466-475.

6. Sano Y, Muto M, Tajiri H, Ohtsu A, Yoshida, S. Optical/digital chromoendoscopy during colonoscopy using narrow-band imaging system. Dig Endo 2005; 17: S43-S48.

7. Muto M, Katada C, Sano Y, Yoshida S. Narrow band imaging: a new diagnostic approach to visualize angiogenesis in superficial neoplasia. Clin Gastroenterol Hepatol 2005; 3(7 Suppl 1): S16-20.

8. Kodashima, S, Fujishiro M. Novel imageenhanced endoscopy with i-scan technology. World J Gastroenterol 2010; 16(9): 1043-9.

9. ASGE Technology Committee, Manfredi MA, Abu Dayyeh BK, Bhat YM, Chauhan SS, Gottlieb $\mathrm{KT}$, et al., Electronic chromoendoscopy. Gastrointest Endosc 2015; 81(2): 249-61.

10. Cammarota G, Ianiro G, Sparano L, La Mura R, Ricci R, Larocca LM, et al. Image-enhanced endoscopy with I-scan technology for the evaluation of duodenal villous patterns. Dig Dis Sci 2013; 58(5):1287-92.

11. Glover B, Patel N, Ashrafian H, Teare J. Diagnostic accuracy of i-scan image enhancement for real-time endoscopic diagnosis of small colorectal polyps: a meta-analysis. Therap $A d v$ Gastroenterol $2018 \quad$ Dec 6; 11:1756284818814948. 